\title{
Sex differences after environmental enrichment and physical exercise in rats when solving a navigation task
}

\author{
V. D. Chamizo ${ }^{1,3}$ • C. A. Rodríguez ${ }^{1,3}$ - J. Sánchez ${ }^{2}$ F. Mármol $^{2}$
}

Published online: 28 October 2015

(C) Psychonomic Society, Inc. 2015

\begin{abstract}
The effects of early environmental enrichment (EE) and voluntary wheel running on the preference for using a landmark or pool geometry when solving a simple spatial task in adult male and female rats were assessed. After weaning, rats were housed in same-sex pairs in enriched or standard cages (EE and control groups) for two and a half months. Then the rats were trained in a triangular-shaped pool to find a hidden platform whose location was defined in terms of these two sources of information, a landmark outside the pool and a particular corner of the pool. As expected, enriched rats reached the platform faster than control animals, and males and females did not differ. Enriched rats also performed better on subsequent test trials without the platform with the cues individually presented (either pool geometry or landmark). However, on a preference test without the platform, a clear sex difference was found: Females spent more time in an area of the pool that corresponded to the landmark, whereas males spent more time in the distinctive corner of the pool. The present EE protocol did not alter females' preference for the landmark cue. The results agree with the claim that environmental enrichment is a consequence of a reduced anxiety response (measured by thigmotaxis) during cognitive testing. A
\end{abstract}

V. D. Chamizo

victoria.diez.chamizo@ub.edu

1 Departament de Psicologia Bàsica, Facultat de Psicologia, Universitat de Barcelona, Barcelona, Spain

2 Unitat de Farmacologia, Facultat de Medicina, Universitat de Barcelona, Barcelona, Spain

3 Institute for Brain, Cognition and Behavior (IR3C), Passeig de la Vall d'Hebron 171, 08035 Barcelona, Spain possible implication of ancestral selection pressures is discussed.

Keywords Environmental enrichment · Voluntary wheel running · Geometry and landmark learning · Thigmotaxis · Sex differences $\cdot$ Natural selection $\cdot$ Morris water-maze $\cdot$ Rats

Research conducted with nonhuman subjects has revealed the important influence of early sensory experience, like environmental enrichment, on brain development and in the capacity for learning in adult life (for reviews, see Nithianantharajah \& Hannan, 2006; Simpson \& Kelly, 2011). One definition of environmental enrichment is a housing condition that 'facilitates enhanced sensory, cognitive and motor stimulation relative to standard housing conditions' (Nithianantharajah \& Hannan, 2006). Environmentally enriched animals perform better on cognitive tests conducted when they are adults, with spatial tasks being frequently employed for this purpose (Harris, D'Eath, \& Healy, 2009; Kolb \& Gibb, 1991; Leggio et al., 2005; Nilsson, Perfilieva, Johansson, Orwar, \& Eriksson, 1999; Pham, Söderström, Winbald, \& Mohammed, 1999). Among spatial tasks, the Morris water navigation task (Morris, 1981) is probably the procedure most widely used to study spatial learning, with the hippocampus identified as a crucial brain area for spatial learning (for reviews, see Knierim \& Hamilton, 2010; Pearce, 2009; Sutherland \& Hamilton, 2004). Similar to EE, enhanced performance, in both humans and animals, is found with physical exercise, like voluntary wheel running in rats (for a review, see Sherwin, 1998). Running has been claimed to increase neurogenesis in the dentate gyrus region (DG) of the hippocampus (Uda, Ishido, Kami, 
\& Masuhara, 2006; van Praag, Christie, Sejnowski, \& Gage 1999a; van Praag, Kempermann, \& Gage 1999b), as does environmental enrichment (Falkenberg, Ernfors, Persson, \& Lindefors, 1992; Kempermann, Kuhn, \& Gage, 1997; Kuhn, Dickinson-Anson, \& Gage, 1996; Nilsson et al., 1999). These findings suggest that enriched and exercised animals could be better prepared than control rats to solve spatial hippocampus-dependent tasks, thus explaining their superior performance while navigating. But a complete explanation of early sensory experience is still a matter of debate. A recent hypothesis suggested by Harris et al. (2009; see also Harris, D'Eath, \& Healy, 2008) to explain these results while measuring thigmotaxis in the Morris pool-swimming close to the wall of the pool-is that enhanced cognition in enriched rodents is an indirect consequence of a reduced anxiety response during cognitive testing (i.e., during a stressful experience) as a result of habituation to novelty - an explanation based on a performance effect. The Morris water navigation task (as many other "cognitive" tasks) induces a strong stress response that is gradually attenuated (Aguilar-Valles et al., 2005). Moreover, it has been observed that environmental enrichment can produce, both in male and female rats, a decrease in the baseline levels of corticosterone and corticotropic stress-responsive hormones regulated by the hypothalamic-pituitary-adrenal (HPA) axis, thus being able to modulate the stress/anxiety response (Belz, Kennell, Czambel, Rubin, \& Rhodes, 2003).

When solving spatial tasks, males and females can rely on different cues (Williams, Barnett, \& Meck, 1990; Williams \& Meck, 1991; see also Hawley, Grissom, Barratt, Conrad, \& Dohanich, 2012; Keeley, Tyndall, Scott, \& Saucier, 2013; Roof \& Stein, 1999; Rodríguez, Torres, Mackintosh, \& Chamizo, 2010; Rodríguez, Chamizo, \& Mackintosh, 2011). Males tend to use geometric information (such as the shape and dimension of the experimental room or specific apparatus), while females use more landmarks, specific objects near the goal. The same claim has been made with humans (Dabbs, Chang, Strong, \& Milun, 1998; Galea \& Kimura, 1993; Jones \& Healy, 2006; Sandstrom, Kaufman, \& Huettel, 1998; Saucier et al., 2002). These findings are in spite of the fact that most reflect a single behavioral dissociation, which perhaps cannot explain the richness and diversity of the psychological and behavioral processes involved in the spatial tasks implicated (Sutherland \& Hamilton, 2004). In the present study we will focus on the work by Rodríguez et al. (2010), with rats and a modified Morris pool.

In the study by Rodríguez et al. (2010, Experiment 2), two groups of adult male and female rats were trained in an unusual triangular pool to find a hidden platform, whose location was defined in terms of two sources of information: a landmark outside the pool and a particular corner of the pool. After acquisition, three test trials were conducted without the platform: a preference test (where the two cues were presented simultaneously) and two single-cue learning tests (where the two cues were presented individually). On the preference test a clear sex difference was found: males spent more time in the distinctive corner of the pool while females spent more time in the area of the pool next to the landmark, even though the single-cue tests revealed that both males and females had learned about the two cues. Moreover, a clear male advantage on geometry learning was also found. The two experiments showed that males and females differed in their preference for using a landmark or the pool geometry when solving a simple spatial task (i.e., a "qualitative" sex difference). An important question to answer is could these qualitative sex differences be changed with experience?

Subsequent work by Rodríguez, Chamizo, and Mackintosh (2013) has shown that the rats' age is related to female task solution strategy. Rodríguez et al. (2013) showed that 30-dayold females behave like adult males, responding to the geometrical shape of the pool rather than the landmark on the preference test. The differences observed in this study were solely in their preferred mode of solution as adults, with juvenile females using the pool geometry in preference to the landmark (for a similar result, see Kanit et al., 2000). Due to the fact that ovariectomized females behaved more like younger than older rats, it was suggested that the explanation of the change in the behaviour of female rats was related to hormonal changes associated with the onset of puberty (for a similar conclusion in humans, see a revision by Voyer, Voyer, \& Bryden, 1995). Could early stimulation (specifically, the combination of environmental enrichment and voluntary wheel running-EE in the present study) alter or modulate the results found by Rodríguez et al. (2010) on the preference test with adult female rats? The first aim of this study is to answer this question. If that is the case, early EE could counteract the effects of the hormonal changes associated with puberty in female rats (Rodríguez et al., 2013).

In the present work, first we focused on raising our animals in same-sex pairs in enriched or standard cages for two and a half months (i.e., EE and control groups, respectively - with an equal number of males and females in each group). Following this, when the rats were approximately 90 days old, they were trained and tested as in Rodríguez et al. (2010, Experiment 2-although with the three test trials counterbalanced). We predicted that enriched rats, both males and females, would always perform better than control animals. But the critical question was would the EE protocol alter the preferences of the adult females, responding to the geometrical shape of the pool rather than the landmark on the preference test? We also chose to follow Harris et al. (2009) and record anxiety measured by thigmotaxis. Finally, after the test trials in the Morris pool all rats were tested in a modified straight corridor for a secondary measure of anxiety. 


\section{Method}

\section{Subjects}

The subjects were 32 naïve Long Evans rats (Rattus norvegicus), 16 males and 16 females from our own colony. They were distributed into two groups ( 8 males and 8 females each group) and housed in same-sex pairs on postnatal Day 22. The groups contained equal numbers of rats from each litter, with the same number of males and females from each litter, thereby providing equal matched pairs for comparison.

All animal treatment and care abided by the principles of the University of Barcelona as well as by the corresponding principles of the European Community (EEC Council Directive 86/609/EEC).

\section{Environmental enrichment (housing conditions)}

Immediately after weaning, the animals were housed in either an enriched or a control cage, and thus were reared under either enriched or standard conditions. The enriched environment consisted of large aluminum cages measuring $45 \times 35 \times 50 \mathrm{~cm}$ and containing a combination of different objects, or "toys." Twenty different combinations were used (for two examples, see Fig. 1, top of the page), with two changes every week (i.e., a new combination after every third and fourth day). These combinations included different objects made of wood, metal, or hard plastic (e.g., tunnels, running wheels, balls of different size) as well as ramps, ropes, and so on. One specific object, a running wheel, was present in all combinations. The control environment consisted of standard methacrylate and aluminum cages measuring $25 \times 15 \times 50 \mathrm{~cm}$, identical to those used for housing the animals in the colony room. The two sets of cages (i.e., enriched and control) were provided with a $2 \mathrm{~cm}$ layer of woodchip bedding and were kept in the same room, although on different racks. The animals were always maintained on ad lib food and water, with a 12:12 hour light-dark cycle. The temperature of the home room was kept at $23{ }^{\circ} \mathrm{C}$, with a relative humidity of $56 \%$.

The total duration of the EE procedure was two and half months. Because brain regions can grow at different rates (Lenroot \& Giedd, 2010), we thought this amount of time was important. During this period all the rats were handled twice a week, coinciding with the change of the combination of objects in the enriched cages. A stereotyped handling procedure for all animals was adopted. A rat was picked up by placing one hand over its shoulders and back, with the fingers lightly but firmly closed under its chin and chest. Once a week (when the cages were cleaned), the two rats in each cage (any cage) were placed in a sink, which was next to the racks, for approximately 2 minutes (the time needed to completely change the woodchip bedding and objects in an enriched cage) and were then placed back into the appropriate cage individually. On the second weekly occasion the rats were handled, the procedure was similar to that just described for EE rats (with the only exception that the woodchip bedding was not changed). Therefore, handling of the animals by an experimenter (who was always the same for each animal) was equated in the two groups. After the enrichment procedure period, the EE animals remained in the large cages, but without objects (with the sole exception of the running wheel), and the control animals stayed in the standard cages. The reason for leaving the running wheel in the cages was to further stimulate the rats development. The behavioral measures began when the animals were 3 months old. After the behavioral measures the rats were sacrificed, and both their cerebral cortex and hippocampus were extracted. Several experiments were conducted with these areas to determine oxidative stress. The results showed that our protocol (environmental enrichment and voluntary wheel running) reduced markers of oxidative stress in the two cerebral regions (Mármol, Rodríguez, Sánchez, \& Chamizo, 2015).

\section{Apparatus}

\section{Circular pool and modified pool}

The Morris water-maze navigation paradigm was a circular swimming pool made of plastic and fiberglass (Rodríguez et al., 2010; Rodríguez et al., 2013). It measured $1.58 \mathrm{~m}$ in diameter, with a depth of $65 \mathrm{~cm}$, and for the experiment it was filled to a depth of $49 \mathrm{~cm}$ with water rendered opaque by the addition of $1 \mathrm{cl} / 1$ of polystyrene latex, a nontoxic substance that does not spoil. The water temperature was maintained at $22 \pm 1^{\circ} \mathrm{C}$. The pool was situated in the middle of a large room and mounted on a wooden platform $43 \mathrm{~cm}$ above the floor. To create the triangular geometry, two acrylic boards forming an angle of $90^{\circ}$ were placed inside the pool on top of platforms at the base which supported them vertically. The boards were $39.5 \mathrm{~cm}$ high, $0.5 \mathrm{~cm}$ thick, and $1.12 \mathrm{~m}$ long. The top of the boards emerged $9.5 \mathrm{~cm}$ above the water surface, which was the same height as the outer wall of the pool. The pool was surrounded by black curtains reaching from the ceiling to the base of the pool and forming a circular enclosure $2.4 \mathrm{~m}$ in diameter. A single object, Landmark X, was suspended from a black false ceiling inside this enclosure, $35 \mathrm{~cm}$ above the surface of the water and with its mid-line directly above the wall of the pool. For all rats, Landmark $\mathrm{X}$ was a ninepin bowling pin (with identical blue and yellow vertical segments), $6 \mathrm{~cm}$ in diameter at the base and $16.5 \mathrm{~cm}$ in height, with the wider part measuring $26 \mathrm{~cm}$ in circumference. The single Landmark X, as well as the point formed by the corner of the pool with a straight wall to the left and the circular base 


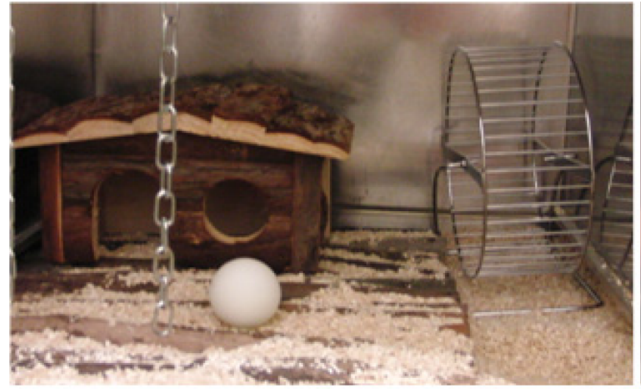

Combination $n^{\circ} 3$

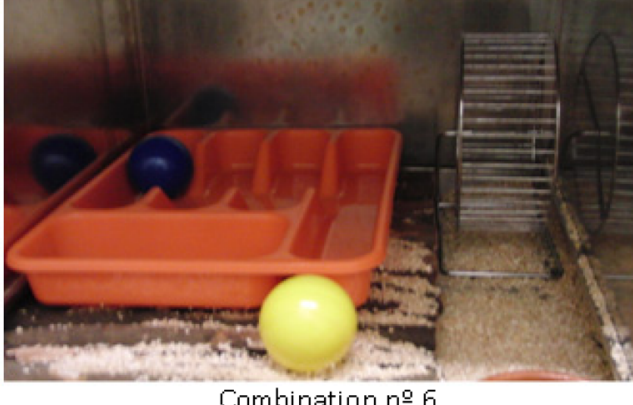

Combination no 6

a

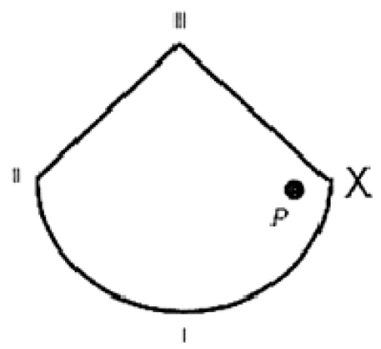

b

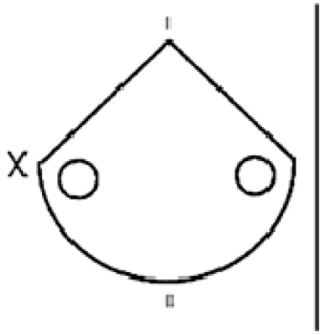

Fig. 1 Top of the page: Two examples of the 20 combinations of the enriched cages used in this study (specifically, Combinations 3 and 6). Bottom of the page: A schematic representation of the pool and the position of the landmark, $\mathrm{X}$, as well as the hidden platform (P). a: for acquisition; $\mathbf{b}$ : for the preference test; $\mathbf{c}$ : for the learning test with the landmark and the circular pool, where the white and the gray circles reflect the target and the control areas, respectively; and $\mathbf{d}$ : for the learning test with the geometry of the pool and without the landmark, where the white and the gray circles reflect the target and the control areas, respectively of the triangle to the right, defined the location of the platform. In order to ensure that the rats used these two sources of information (the landmark and the triangular shaped pool) to locate the platform, rather than any inadvertently remaining static room cues (like noises from pipes and air conditioning), the landmark, the two boards, and the platform were semirandomly rotated with respect to the room $\left(90^{\circ}, 180^{\circ}\right.$, $270^{\circ}$, or $360^{\circ}$ ) with the restriction that all four positions of the room were used each day. A closed-circuit video camera with a wide-angle lens was mounted $1.75 \mathrm{~m}$ above the center of the pool inside the false ceiling, relaying to recording equipment in an adjacent room. A circular platform $11 \mathrm{~cm}$ in diameter and made of transparent Perspex was mounted on a rod and base which was placed $38 \mathrm{~cm}$ from the point formed by the corner of the pool with a straight wall to the left and the circular base of the triangle to the right, on a line that bisected the center of the pool, with its top $1 \mathrm{~cm}$ below the surface of the water. The hidden Platform, P, Landmark X, and the geometry of the pool were situated as shown in Fig. 1a.

\section{Modified straight corridor}

The modified straight corridor consisted of a wooden uncovered box of $22.5 \mathrm{~cm}$ wide, $30 \mathrm{~cm}$ high, and $2.22 \mathrm{~m}$ long, which for the present purposes was divided into two parts: the start box, $25 \mathrm{~cm}$ long, which was just outside the perimeter of the pool and therefore in a darker light, and the rest of the corridor. It was located above the Morris pool, at a distance of about $22 \mathrm{~cm}$ above the water level (i.e., relatively close to the lighting system in order to produce some distress), in a way that the time to leave the start box was automatically registered by the computer tracking system.

\section{Procedure}

\section{Circular pool and modified pool}

There were three types of trials: pretraining, training, and test trials. Pretraining consisted of placing a rat into the circular 
pool without the landmark or boards but with the hidden platform present. The rat was given $120 \mathrm{~s}$ to find the platform, and once it had been found, the rat was allowed to stay on it for 30 s. If the rat had not found the platform within $120 \mathrm{~s}$, it was picked up, placed on it and left there for $30 \mathrm{~s}$. The platform was moved from one trial to the next, and the rat was placed in the pool in a different location on each trial; rats were placed equally often on the same or opposite side of the pool from the platform, and with the platform to the right or the left of where the rat was placed. Rats performed five of these pretraining trials over 2 days, with two trials on Day 1 and three on Day 2. They were run in squads of eight, and spent the intertrial interval (ITI) in small individual compartments. The time (in seconds) to reach the hidden platform, the distance traveled (in $\mathrm{mm}$ ), and the swim speed were automatically recorded.

The procedure for training was similar to that of pretraining, although with three exceptions. The landmark, $\mathrm{X}$ (the ninepin), was always present, as were the two boards forming the triangular pool, as shown in Fig. 1a. As in pretraining, the rat was placed in the pool in a different location on each trial, equating as far as possible the number of occasions with the platform to the right, to the left, or in front of where the rat was placed (at positions I, II, and III of the previous figure). Rats received eight trials per day over 5 days (a total of 40 trials). These trials had an ITI of 8 to 10 minutes, and the platform, landmark, and triangular geometry were rotated between trials. Finally, the time (in seconds) to reach the hidden platform was automatically recorded.

After the training phase, there were 3 consecutive test days, each starting with eight training trials (identical to the training phase), followed by one test trial without the platform. The test trials were counterbalanced and always lasted $60 \mathrm{~s}$. On one test trial, a conflict test, the two sources of information (the landmark and the triangular pool) were presented $180^{\circ}$ apart. The amount of time that the rat spent in two different but identically sized areas (i.e., one in front of the landmark and the second one in front of the distinctive corner of the pool, as shown in Fig. 1b), was recorded. In the other two test trials, learning tests, the rats were tested either in the circular pool with the landmark or in the triangular pool with no landmark - as shown in Fig. 1 (c and d, respectively). The amount of time that the rats spent in two different but identically sized areas (i.e., one close to either the landmark or the distinctive corner of the pool and the second one $180^{\circ}$ apart, a control area) was recorded in each test. The reason for measuring the time spent in the control area as well as the target area (c and d in Fig. 1-where the white and the gray circles reflect the target and the control areas, respectively) was to check whether, on the geometry test (i.e., in the absence of the landmark), rats could discriminate between the two similar corners of the triangle, and whether, on the landmark test (i.e., in the absence of the triangular pool), they were simply swimming in a circle at a certain distance from the wall of the pool. On test trials, each rat was placed in the pool from one specific position-at I and II only, as shown in Fig. 1 (b, c, and d), and the two starting positions, I and II, were equally used by the two groups (EE and Control).

\section{Thigmotaxis}

Anxiety was measured in terms of thigmotaxis (i.e., wall hugging, rats' natural proclivity to stay near the perimeters of a novel environment; see Treit \& Fundytus, 1988). Specifically, as Harris et al. (2009) did, the proportion of time that a rat spent swimming within $15 \mathrm{~cm}$ of the wall of the maze was recorded. Such a measure was used on all escape trials (i.e., during pretraining in the circular pool, throughout training, and on the escape trials conducted on the three test days in the triangular pool) as well as in the test trials. All measures were automatically registered by a computer. An alpha level of .05 was adopted for all statistical analyses. Only significant results are presented.

Following the pool, all rats were tested in a modified straight corridor for a complementary behavioral anxiety measure.

\section{Modified straight corridor}

A single trial was carried out over three consecutive days. Each trial consisted of placing an animal in the start box for $60 \mathrm{~s}$-with the doors of the corridor always open. The time taken by the animal to abandon the start box (i.e., the latency) was automatically registered. The number of defecations during the whole minute was also measured.

\section{Results $^{1}$}

Figure 2a shows that the latencies for finding the platform for the two groups (EE and Control - males and females, respectively) decreased over the course of the five initial pretraining trials. A repeated measures ANOVA conducted on these data taking into account the variables trials (1-5), Enrichment group (EE, Control), and sex showed that the only significant variable was trials, $F(4,112)=20.19, p<.01, \eta_{\mathrm{p}}^{2}=0.42$. No other main effect or interaction was significant $(F \mathrm{~s}<4.0)$. There was no difference between the rats in latency to find the platform over the five trials of pretraining. All animals reduced their latencies as pretraining trials progressed, probably due to a reduced thigmotaxis.

Figure $2 \mathrm{~b}$ shows that the mean distance traveled by the animals in reaching the platform decreased over the course of the five pretraining trials. A repeated measures ANOVA

\footnotetext{
${ }^{1}$ Unfortunately, the variable litter could not be taken into account in the analysis, as this information was lost due to human error.
} 

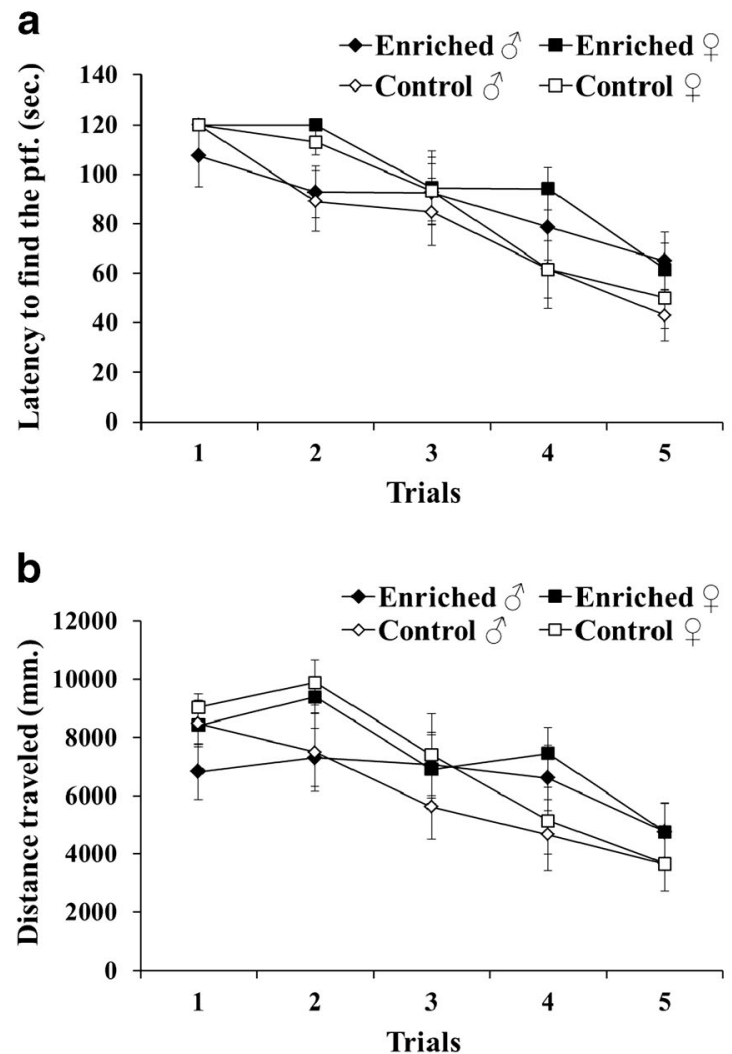

Fig. 2 Results for the two groups (Enriched and Control-males and females, respectively) in the circular pool, during the pretraining trials (Trials 1-5). a: Mean escape latencies. b: Distance traveled. c: Swim

conducted on these data taking into account the same variables (trials, group, sex) showed that the only significant variable was trials, $F(4,112)=13.41, p<.01, \eta_{\mathrm{p}}^{2}=0.32$. No other main effect or interaction was significant $\left(F_{\mathrm{s}}<3.5\right)$. There was no difference between the rats in the distance swum to find the platform over the five trials of pretraining. All animals reduced their distance swum as pretraining trials progressed, probably due to a reduced thigmotaxis.

Figure $2 \mathrm{c}$ shows the swimming speed of the animals over the course of the five pretraining trials. A repeated measures ANOVA conducted on these data taking into account the same variables (trials, group, sex) showed that the only significant variable was trials, $F(4,112)=2.69, p=0.035, \eta_{p}^{2}=0.09$. No other main effect or interaction was significant $(F \mathbf{S}<1.0)$. In general (mainly on trials 2, 4, and 5), all animals maintained the same swimming speed as pretraining trials progressed.

Finally, Fig. $2 \mathrm{~d}$ shows that the time spent swimming by the rats in the thigmotaxis area (i.e. within $15 \mathrm{~cm}$ of the wall) decreased over the course of the five pretraining trials. A repeated measures ANOVA conducted on these data taking into account the same variables (trials, group, sex) showed that the only significant variable was trials, $F(4,112)=44.36, p<.01$, $\eta_{\mathrm{p}}^{2}=0.61$. The time spent swimming in the thigmotaxis area declined with repeated trials in all rats. No other main effect or interaction was significant $\left(F_{\mathrm{S}}<3.0\right)$. There was no difference
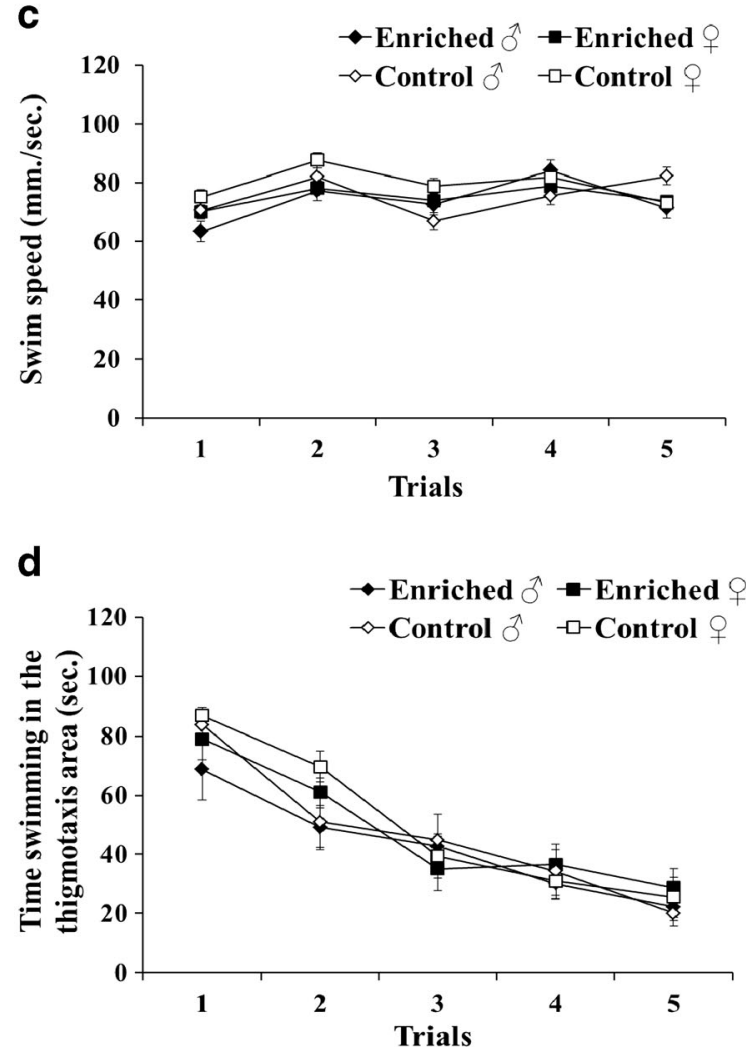

speed. d: Mean time spent swimming in the thigmotaxis area (i.e., within $15 \mathrm{~cm}$ of the wall of the pool). (Error bars always denote standard error of means.)

between the rats in the time spent swimming in the thigmotaxis area over the five trials of pretraining. All animals reduced their time swimming in the thigmotaxis area as pretraining trials progressed.

In conclusion, no beneficial effects of environmental enrichment were found in the different measures registered during the pretraining trials, neither in males nor in females, and enriched and control rats were equally thigmotactic.

Figure $3 \mathrm{a}$ shows that the latencies for finding the platform for the two groups (EE and Control) also decreased over the course of the training days and on the escape trials of the test days in the modified Morris pool. A repeated measures ANOVA conducted on the training days taking into account the variables days (1-5), group (EE, Control), and sex showed that the variables days, $F(4,112)=101.62, p<.01$, $\eta_{\mathrm{p}}^{2}=0.78$, and group, $F(1,28)=28.71, p<.01, \eta_{\mathrm{p}}^{2}=0.51$, were significant, as well as the interaction days $\times$ group, $F(4$, $112)=2.70, p=.034, \eta_{\mathrm{p}}^{2}=0.88$. No other main effect or interaction was significant $\left(F_{\mathrm{S}}<1.0\right)$. The analysis of the interaction days $\times$ group showed that the groups differed in all the training days, $F_{\mathrm{s}}(1,30)=10.16, p=.003, \eta_{\mathrm{p}}^{2}=0.25$; $8.39, p=.007, \eta_{\mathrm{p}}^{2}=0.22,9.84, p=.004, \eta_{\mathrm{p}}^{2}=0.25,5.72, p$ $=.023, \eta_{\mathrm{p}}^{2}=0.16$, and 17.78, $p<.01, \eta_{\mathrm{p}}^{2}=0.37$, Days 1 through 5 , respectively. A repeated measures ANOVA conducted on the escape trials of the three test days, taking into 


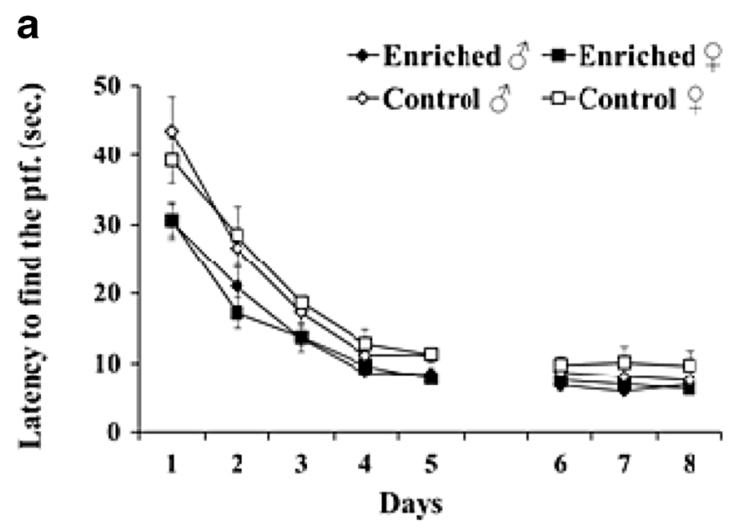

b

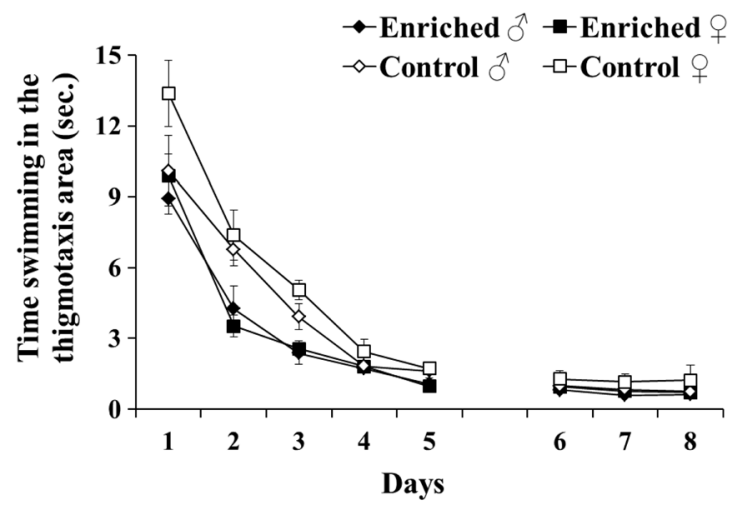

Fig. 3 Results for the two groups (Enriched and Control-males and females, respectively) in the modified Morris pool. a: Mean escape latencies during the training (Days 1-5) and test phases (Days 6-8). b: Mean time spent swimming in the thigmotaxis area (i.e., within $15 \mathrm{~cm}$ of the wall of the pool) during the training days (Days 1-5) and on the

account the variables days (6-8), group (EE, Control), and sex, revealed that the only variable significant was group, $F(1,28)=5.31, p=.029, \eta_{\mathrm{p}}^{2}=0.16$. No other main effect or interaction was significant $\left(F_{\mathrm{S}}<2.0\right)$. The results of Fig. 3a show a beneficial effect of environmental enrichment and no sex differences: both during the training phase and on the escape trials of the test phase enriched rats reached the platform faster than control animals.

Figure $3 \mathrm{~b}$ shows that the time spent swimming in the thigmotaxis area for the two groups (EE and Control) decreased over the course of the training days and on the escape trials of the test days. A repeated measures ANOVA conducted on the training days taking into account the variables days (1-5), group (EE, Control), and sex, showed that the variables days, $F(4,112)=115.75, p<.01, \eta^{2} \mathrm{p}=0.80$, and group, $F(1,28)=$ $31.35, p<.01, \eta_{\mathrm{p}}^{2}=0.53$, were significant, as well as the interaction days $\times$ group, $F(4,112)=2.91, p=.025, \eta_{\mathrm{p}}^{2}=$ 0.09 . No other main effect or interaction was significant $\left(F_{\mathrm{S}}<\right.$ $4.5)$, although the variable sex just failed to reach criterion ( $p=$ $.05)$. The analysis of the interaction days $\mathrm{x}$ group showed that the groups differed on Days 2, 3 and 5, $F_{\mathrm{s}}(1,30)=15.28, p<$
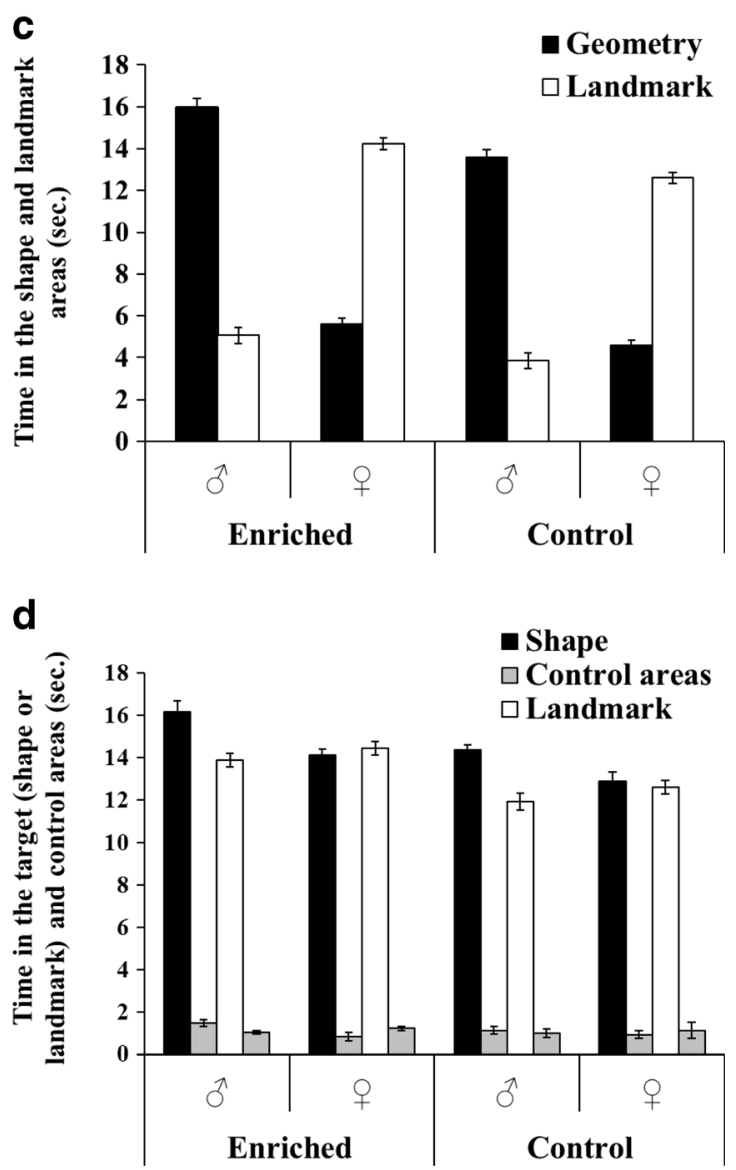

escape trials of the test days (Days 6-8). c: Mean time spent in the two recording areas (geometry and landmark) during the preference test trial. d: Mean time spent in the two recording areas (target and control) during the two learning test trials (geometry and landmark). (Error bars always denote standard error of means.)

$.01, \eta_{\mathrm{p}}^{2}=0.34,20.19, p<.01, \eta_{\mathrm{p}}^{2}=0.40$, and 13.68, $p=.01$, $\eta_{\mathrm{p}}^{2}=0.31$, respectively. In addition, the two groups were close to being different on Day $1(p=.06)$. A repeated measures ANOVA conducted on the time spent swimming in the thigmotaxis area during the escape trials of the three test days, taking into account the variables days (6-8), group (EE, Control), and sex, showed that no variable or interaction was significant $\left(F_{\mathrm{S}}<2.5\right)$. The results of Fig. 3b show that the time spent swimming in the thigmotaxis area declined with repeated trials during the training days only, when enriched rats (both males and females) were less thigmotactic than control animals. All rats were equally thigmotactic on the test days.

Figure $3 \mathrm{c}$ shows the time spent in the two recording areas (i.e., landmark area and geometry area) by the two groups on the preference test trial. A repeated measures ANOVA conducted on these data taking into account the variables group (EE, Control), type of cue tested (landmark, geometry), and sex revealed that the variable group was significant, $F(1,28)=$ $55.07, p<.01, \eta_{\mathrm{p}}^{2}=0.66$, showing that $\mathrm{EE}$ rats spent more time swimming in the two recording areas than Control animals. The variable cue, $F(1,28)=14.04, p=.001, \eta_{\mathrm{p}}^{2}=0.33$, 
was also significant (showing that rats spent more time swimming in the geometry area than in the landmark area), as well as the interaction cue $\times \operatorname{sex}, F(1,28)=1252.41, p<.01, \eta^{2}=$ 0.98 . No other main effect or interaction was significant $\left(F_{\mathrm{S}}<\right.$ 3.0). The analysis of the interaction cue $x$ sex showed that males and females differed in the amount of time spent both in the landmark and in the geometry areas, $F \mathrm{~s}(1,30)=422.53$, $p<.01, \eta_{\mathrm{p}}^{2}=0.93$, and $474.39, p<.01, \eta_{\mathrm{p}}^{2}=0.94$, respectively. While male rats spent more time in the geometry area than in the landmark area, $F(1,15)=589.22, p<.01, \eta_{\mathrm{p}}^{2}=$ 0.97 , the reverse was true for females, $F(1,15)=642.22, p<$ $.01, \eta_{\mathrm{p}}^{2}=0.98$. The results of Fig. $3 \mathrm{c}$ seem to indicate a beneficial motor effect of environmental enrichment because both cues, the preferred one (geometry for males, landmark for females) and the less preferred cue (landmark for males, geometry for females) were affected: Enriched rats spent more time both in the distinctive corner of the pool and in the area of the pool next to the landmark than Control animals. A clear sex difference was also found: males tended to use the geometric information given by the geometry of the pool while female rats relied more on the landmark (thus replicating the results by Rodríguez et al., 2010, Experiment 2). Therefore, the present EE protocol did not alter females' preference for the landmark cue.

Figure $3 \mathrm{~d}$ shows the time spent in the two recording areas (i.e., the target area and the control area) by the two groups during the two single cue test trials (i.e., geometry and landmark). Student's $t$ tests were used to compare rats' performance in each target area with the corresponding control area. On both kinds of test trial, males and females in both groups spent a significantly longer time in the target than in the control area, minimum $t(7)=23.67$, and all $p \mathrm{~s}<.01$, minimum $d=$ 0.05 . This implies that males and females in the two groups (EE and Control) had learned about both the landmark and the correct corner. A repeated measures ANOVA conducted on the time spent in the target area on landmark and geometry tests taking into account the variables group (EE, Control), type of cue tested (geometry, landmark), and sex showed that the variable group was significant, $F(1,28)=45.57, p<.01$, $\eta_{\mathrm{p}}^{2}=0.62$, showing that EE rats spent more time swimming in the two target areas than Control animals. The variable cue, $F(1,28)=18.61, p<.01, \eta_{\mathrm{p}}^{2}=0.40$. was also significant (showing that rats spent more time swimming in the geometry area than in the landmark area), as well as sex, $F(1,28)=5.14$, $p=.031, \eta_{\mathrm{p}}^{2}=0.15$, showing that males performed better than females, and the interaction cue $\times \operatorname{sex}, F(1,28)=19.62, p<$ $.01, \eta_{\mathrm{p}}^{2}=0.41$. No other main effect or interaction was significant $\left(F_{\mathrm{S}}<1.0\right)$. The analysis of the interaction cue $\times$ sex revealed that males performed better on the geometry test than females, $F(1,30)=13.81, p=.001, \eta_{\mathrm{p}}^{2}=0.31$, while the two sexes did not differ on the landmark test $\left(F_{\mathrm{S}}<2.0\right)$. In addition, while males spent more time searching for the platform in the target area on the geometry test than on the landmark test, $F(1,15)=40.35, p<.01, \eta^{2}{ }_{\mathrm{p}}=0.73$, females did not differ on the two tests $\left(F_{\mathbf{S}}<0.5\right)$. Complementarily, a repeated measures ANOVA conducted on the time spent in the control area on landmark and geometry tests taking into account the variables group (EE, Control), type of cue tested (geometry, landmark), and sex showed that the interaction cue $\times$ sex approached criterion, $F(1,28)=4.19, p=.05, \eta_{p}^{2}=0.13$. No other main effect or interaction was significant $\left(F_{\mathrm{S}}<\right.$ 1.0). The results of Fig. $3 \mathrm{~d}$ firstly indicate that both males and females had learned to find the platform using both sources of information (i.e., the landmark and the geometry of the pool). In addition, a clear male advantage was found on geometry learning (thus replicating the results by Rodríguez et al., 2010, Experiment 2). A clear beneficial effect of environmental enrichment was also obtained: Enriched rats performed better in the target area on landmark and geometry tests than Control animals.

Figure 4 shows the time spent swimming in the thigmotaxis area for the two groups (EE and Control - males and females, respectively) on the test trials. A repeated measures ANOVA conducted on these data taking into account the variables tests (preference, geometry learning, landmark learning), group (EE, Control), and sex (male, female), showed that only the variable group was significant, $F(1,28)=6.70, p=.015, \eta_{p}^{2}=$ 0.19 , with enriched rats (both males and females) being less thigmotactic than control animals. No other main effect or interaction was significant $(F \mathrm{~s}<3.0)$, although the variable tests was close $(p=.08)$.

Figure 5 (top panel) shows the latencies to leave the start box by males and females of the two groups (EE and Control) during the three trials in the modified straight corridor. An ANOVA conducted on these data taking into account the variables trials (1-3), group (EE, Control), and sex showed that the variables trials, $F(2,56)=53.95, p<.001, \eta_{\mathrm{p}}^{2}=0.66$, group, $F(1,28)=15.75, p<.001, \eta_{\mathrm{p}}^{2}=0.36$, and sex, $F(1,28)$ $=34.53, p<.001, \eta_{\mathrm{p}}^{2}=0.55$, were significant, as well as the interactions trials $\times$ group, $F(2,56)=4.23, p=.019, \eta_{\mathrm{p}}^{2}=$ 0.13 , and trials $\times \operatorname{sex}, F(2,56)=10.07, p<.001, \eta_{\mathrm{p}}^{2}=0.27$. No other main effect or interaction was significant $\left(F_{\mathrm{S}}<1.0\right)$. The analysis of the interaction trials $\times$ group showed that the groups differed on Trials 1 and 2 only, $F_{\mathrm{s}}(1,30)=5.83, p=$ $.022, \eta_{\mathrm{p}}^{2}=0.16$, and 5.46, $p=.026, \eta_{\mathrm{p}}^{2}=0.15$, respectively, reflecting that animals in the EE group took less time to leave the start box than animals in the Control group. The analysis of the interaction trials $\times$ sex showed that males and females differed on the three trials, $F_{\mathrm{s}}(1,30)$ $=17.91, p<.001, \eta_{\mathrm{p}}^{2}=0.37,13.34, p=.001, \eta_{\mathrm{p}}^{2}=$ 0.31 , and 9.12 , Trials 1 through 3 , respectively, reflecting that female rats took less time to leave the start box than male rats. These analyses suggest that enriched rats (mainly females) were less anxious/fearful in response to a novel environment, thus showing more exploratory behavior than control animals. 


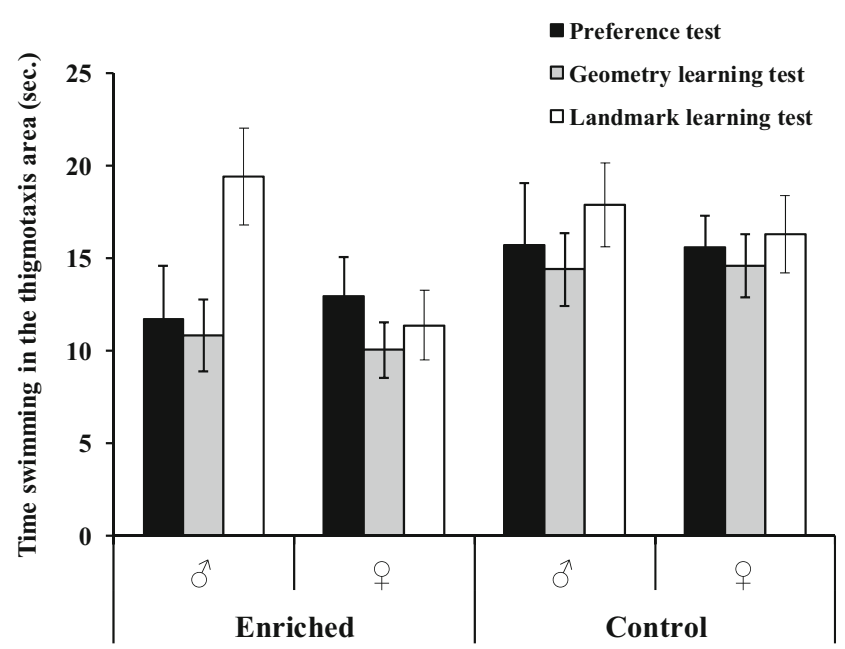

Fig. 4 Results for the two groups (Enriched and Control-males and females, respectively), during the three test trials (preference, geometry learning, landmark learning). Mean time spent swimming in the thigmotaxis area (i.e., within $15 \mathrm{~cm}$ of the wall of the pool). (Error bars always denote standard error of means.)

Figure 5 (bottom panel) shows the mean number of defecations made by the groups during the three trials. An ANOVA conducted on these data taking into account the variables trials (1-3), group (EE, Control), and sex revealed that
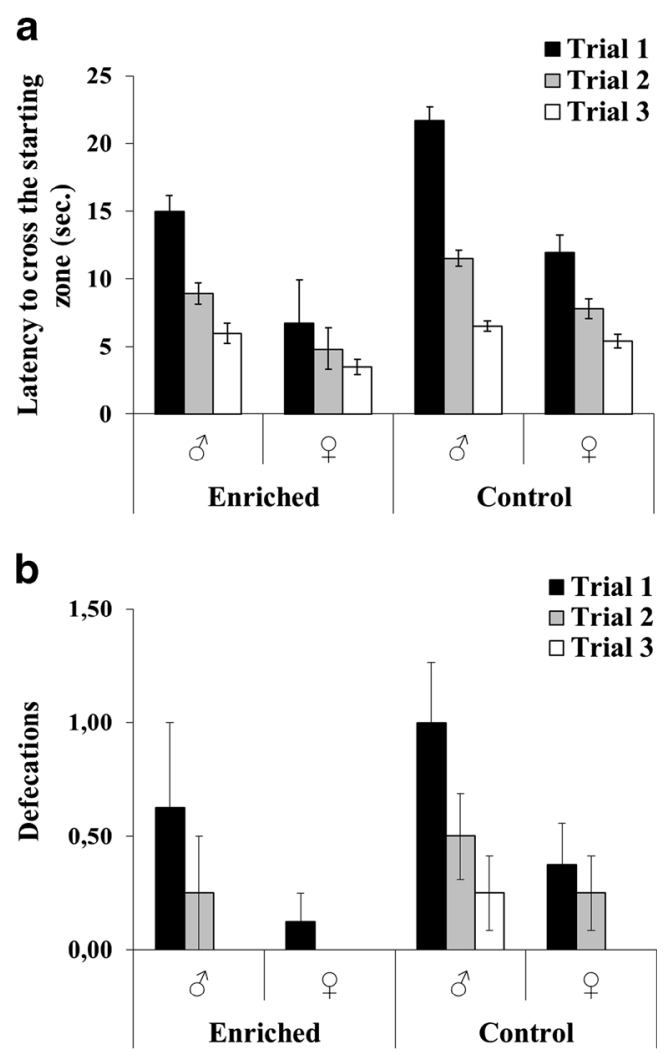

Fig. 5 Results for the two groups (Enriched and Control-males and females, respectively) in the modified straight corridor during the three trials. Top panel: Mean latency to leave the start box. Bottom panel: Mean number of defecations. (Error bars always denote standard error of means.) the variables trials, $F(2,56)=8.26, p=.001, \eta_{\mathrm{p}}^{2}=0.23$, and sex, $F(1,28)=5.94, p=.021, \eta_{\mathrm{p}}^{2}=0.18$, were significant, reflecting that as trials progressed the number of defecations was reduced in all animals. Thus, the visual difference observed in Fig. 5 between EE animals and Control rats (showing that EE rats defecated less than Control animals) did not reach statistical significance. Female rats defecated less than male rats, suggesting that they were less anxious/fearful. No other main effect or interaction was significant $\left(F_{\mathrm{S}}<3.5\right)$.

\section{General discussion}

The results from the five pretraining trials (in the absence of both the landmark and the triangular-shaped pool) revealed that $\mathrm{EE}$ rats achieved neither shorter latencies nor shorter path lengths in finding the platform, compared with Control animals (both males and females). Consequently, EE and Control rats did not differ in swim speed. Because the time spent swimming in the thigmotaxis area declined with repeated trials, we conclude that all rats improved their performance as behavioral anxiety (i.e., thigmotaxis) decreased. Therefore, no beneficial effects of early enriched environmental experience in combination with voluntary wheel running were found in the different measures registered during pretraining. However on training trials (when the platform was located in one particular corner of the triangular-shaped pool, next to a landmark situated outside the pool), a clear beneficial effect of early stimulation (i.e., environmental enrichment and voluntary wheel running) was found, as expected: both during the training phase and on the escape trials of the test phase EE rats reached the platform faster than Control animals (Harris et al., 2009; Leggio et al., 2005; Nilsson et al., 1999; Pham et al., 1999). Because EE and Control animals also differed in terms of thigmotaxis during training, it is possible that the superior performance of EE rats reveals the differential behavioral anxiety in the two groups while learning the task, as suggested by Harris et al. (2009). There was no difference between males and females in their initial measures of learning to find the submerged platform in the Morris pool-either during pretraining or during subsequent training. This suggests that females were no more likely than males to spend time exploring the pool rather than swimming directly to the platform (as in Rodríguez et al., 2010).

Following training, the time spent swimming in the thigmotaxis area on escape trials stabilized. The two groups (EE and Control animals) were equally thigmotactic on the escape trials of the test days. On test trials EE rats performed better than Control animals, although this claim deserves further explanation. On the preference test the results seem to indicate that the present protocol resulted in enhanced motor activity: EE rats spent more time swimming in the two recording areas than Control animals. Moreover, a qualitative sex difference 
was found: males tended to use the geometric information given by the pool geometry while female rats relied more on the landmark (as in Rodríguez et al., 2010). Early enriched environmental experience in combination with voluntary wheel running could not counteract or modulate the effects of the hormonal changes associated with puberty in female rats (Rodríguez et al., 2013; see also Kanit et al., 2000). Therefore, we have to conclude that the present EE protocol could not alter adult females' preference for the landmark cue.

When the two cues were tested individually, the results in the two groups showed that both males and females had learned to find the platform using the two sources of information (i.e., the landmark and one specific corner of the pool). In these tests, a clear beneficial effect of the present protocol was found: EE rats spent more time swimming in the target areas than Control animals. This result suggests that early enriched environmental experience in combination with voluntary wheel running facilitates what the rats learn about the two cues (but not how to use them when adults). Moreover, males in both groups performed better on the geometry test (i.e., in the absence of the landmark) than on the landmark test (i.e., in the absence of the triangular pool), and males in the two groups also spent more time searching for the platform in the target area on the geometry test than did females, while males and females did not differ on the landmark test. Therefore, a clear male advantage on geometry learning was found (as in Rodríguez et al., 2010). In conclusion, the results of the test trials suggest that the beneficial effects of environmental enrichment in combination with voluntary wheel running seem to affect the rats' performance in quantitative ways but not qualitatively (i.e., the preferences of adult male and female rats were not altered).

Interestingly, on test trials, EE and Control animals differed in the time spent swimming in the thigmotaxis area. Enriched rats (both males and females) were less thigmotactic than control animals. This finding was surprising, considering that the two groups were equally thigmotactic on the escape trials of these days. What is the explanation for this result? The reason, it could be argued, is that on the test trials (but not on the escape trials) the animals were in a novel situation, due to the absence of the platform. The enhanced cognition of EE rats (mainly when testing geometry, and landmark learning) could reflect an indirect consequence of a reduced anxiety response as a result of habituation to novelty, as suggested by Harris et al. (2009). The final results in the modified straight corridor, a novel environment - where less fearful/anxious behavior was exhibited by EE rats than by Control animals when latency to leave the start box was measured-agrees with this suggestion. Moreover, in the modified straight corridor (unlike in the Morris pool), female rats exhibited less fearful/anxious behavior than male rats-a result that has often been reported in classical studies of open field behavior (Archer, 1975; Broadhurst, 1975; Gray, 1971). This finding was supported with the measurement of defecation.

Considering the history of our rats (Rattus norvegicus), which shows huge birthrates and that parental care is provided by females only (Parker, 1990), the results of the present test trials, specifically those of the preference test, seem consistent with the argument that males and females of many mammalian species have evolved different strategies for spatial navigation and search due to a selective pressure (Ecuyer-Dab \& Roberts, 2004; Jones, Braithwaite, \& Healy, 2003). In the literature of spatial abilities, one explanation stands out: the range size hypothesis (Eals \& Silverman, 1994; Galea, Kavaliers, Ossenkopp, Innes, \& Hargreaves, 1994; Gaulin \& Fitzgerald, 1986; Langley, 1994). The crucial idea in this hypothesis is that sex differences in task performance have arisen from a process of natural selection: a difference in range expansion between males and females is associated with a difference in spatial cognition. In people, that difference arises largely because men hunted and women gathered (see Silverman, Choi, \& Peters, 2007); in other animals the most frequent explanation refers to a polygynous mating system requiring males that mate with more than one female in a single breeding season to have larger ranges (for reviews, see Halpern, 2012; Jones et al., 2003; Mackintosh, 2011). Support for the range size hypothesis among humans has been indirect until recently (Vashro \& Cashdan, 2015).

Environmental enrichment and physical exercise undoubtedly improve the welfare of caged animals. More importantly, EE protocols and physical exercise can produce dramatic effects at the behavioral, neuroanatomical, and cellular levels in many species (Nithianantharajah \& Hannan, 2006; Simpson $\&$ Kelly, 2011), although the exact variables, manipulations, and mechanisms implicated are still a matter of debate. For example, Harris et al. (2009) have shown that enhanced cognition in enriched rodents can be an indirect consequence of a reduced anxiety response during cognitive testing as a result of habituation to novelty. Our results support this claim. But the present research goes beyond the work in this literature by showing that enhanced cognition in enriched rodents may also depend on ancestral selection pressures related to sex.

Author note This research was supported by grants from the Spanish Ministerio de Ciencia e Innovación (Refs. PSI2010-20424 and PSI201347430-P) to V.D.C.

\section{References}

Aguilar-Valles, A., Sánchez, E., de Gortari, P., Balderas, I., RamírezAmaya, V., Bermúdez-Rattoni, F., \& Joseph-Bravo, P. (2005). Analysis of the stress response in rats trained in the water-maze: Differential expression of corticotrophin-releasing hormone, 
CRHR1, glucocorticoid receptors and brain-derived neurotrophic factor in limbic regions. Neuroendocrinology, 82, 306-319.

Archer, J. (1975). Rodent sex differences in emotional and related behavior. Behavioral Biology, 14, 451-479.

Belz, E. E., Kennell, J. S., Czambel, R. K., Rubin, R. T., \& Rhodes, M. E. (2003). Environmental enrichment lowers stress-responsive hormones in singly housed male and female rats. Pharmacology, Biochemistry and Behavior, 76, 481-486.

Broadhurst, P. L. (1975). The Maudsley reactive and nonreactive strains of rats: A survey. Behavior Genetics, 5, 299-319.

Dabbs, J. M., Jr., Chang, E. L., Strong, R. A., \& Milun, R. (1998). Spatial ability, navigation strategy, and geographic knowledge among men and women. Evolution and Human Behavior, 19, 89-98.

Eals, M., \& Silverman, I. (1994). The hunter-gatherer theory of spatial sex differences: Proximate factors mediating the female advantage in recall of object arrays. Ethology and Sociobiology, 15, 95-105.

Ecuyer-Dab, I., \& Roberts, M. (2004). Have sex differences in spatial ability evolved from male competition for mating and female concern for survival? Cognition, 91, 221-257.

Falkenberg, T., Ernfors, P., Persson, H., \& Lindefors, N. (1992). Cortical transsynaptic activation of tyrosine kinase receptor trkB messenger RNA expression in rat hippocampus. Neuroscience, 51, 883-889.

Galea, L., Kavaliers, M., Ossenkopp, K.-P., Innes, D., \& Hargreaves, E. (1994). Sexually dimorphic spatial learning varies seasonally in two populations of deer mice. Brain Research, 635, 18-26.

Galea, L. A. M., \& Kimura, D. (1993). Sex differences in route learning. Personality and Individual Differences, 14, 53-65.

Gaulin, S., \& Fitzgerald, R. W. (1986). Sex differences in spatial ability: An evolutionary hypothesis and test. American Naturalist, 127, 74 88.

Gray, J. A. (1971). The psychology of fear and stress. London: World University Library.

Halpern, D. F. (2012). Sex differences in cognitive abilities (4th ed.). Hillsdale: Erlbaum.

Harris, A. P., D'Eath, R. B., \& Healy, S. D. (2008). Sex differences, or not, in spatial cognition in albino rats: Acute stress is the key. Animal Behavior, 76, 1579-1589.

Harris, A. P., D'Eath, R. B., \& Healy, S. D. (2009). Environmental enrichment enhances spatial cognition in rats by reducing thigmotaxis (wall hugging) during testing. Animal Behavior, 77, 1459-1464.

Hawley, W. R., Grissom, E. M., Barratt, H. E., Conrad, T. S., \& Dohanich, G. P. (2012). The effects of biological sex and gonadal hormones on learning strategy in adult rats. Physiology and Behavior, 105, 1014 1020.

Jones, C. M., Braithwaite, V. A., \& Healy, S. (2003). The evolution of sex differences in spatial ability. Behavioral Neuroscience, 117, 403411.

Jones, C. M., \& Healy, S. D. (2006). Differences in cue use and spatial memory in men and women. Proceedings of the Royal Society B, $273,2241-2247$.

Kanit, L., Taskiran, D., Yilmaz, Ö. A., Balkan, B., Demirgören, S., Furedy, J. J., \& Pögün, S. (2000). Sexually dimorphic cognitive style in rats emerges after puberty. Brain Research Bulletin, 52, 243-248.

Knierim, J. J., \& Hamilton, D. A. (2010). Framing spatial cognition: Neural representations of proximal and distal frames of reference and their roles in navigation. Physiological Reviews, 92, 12451279.

Keeley, R. J., Tyndall, A. V., Scott, G. A., \& Saucier, D. M. (2013). Sex difference in cue strategy in a modified version of the Morris water task: Correlations between brain and behavior. PLoS ONE, 8(7), e69727.

Kempermann, G., Kuhn, H. G., \& Gage, F. H. (1997). Genetic influence on neurogenesis in the dentate gyrus of adult mice. Proceedings of the National Academy of Sciences of the United States of America, 94(19), 10409-10414.
Kolb, B., \& Gibb, R. (1991). Environmental enrichment and cortical injury: Behavioral and anatomical consequences of frontal cortex lesions. Cerebral Cortex, 1, 189-198.

Kuhn, H. G., Dickinson-Anson, H., \& Gage, F. H. (1996). Neurogenesis in the dentate gyrus of the adult rat: Age-related decrease of neuronal progenitor proliferation. The Journal of Neuroscience: The Official Journal of the Society for Neuroscience, 16, 2027-2033.

Langley, C. M. (1994). Spatial memory in the desert kangaroo rat (Dipodomys deserti). Journal of Comparative Psychology, 108, 3 14.

Leggio, M. G., Mandolesi, L., Federico, F., Spirito, F., Ricci, B., Gelfo, F., \& Petrosini, L. (2005). Environmental enrichment promotes improved spatial abilities and enhanced dendritic growth in the rat. Behavioural Brain Research, 163, 78-90.

Lenroot, R. K., \& Giedd, J. N. (2010). Sex differences in the adolescent brain. Brain and Cognition, 72, 46-55.

Mackintosh, N. J. (2011). IQ and human intelligence (2nd ed.). Oxford: Oxford University Press.

Mármol, F., Rodríguez, C. A., Sánchez, J., \& Chamizo, V. D. (2015). Anti-oxidative effects produced by environmental enrichment in hippocampal and cerebral cortex of male and female rats. Brain Research, 1613, 120-129.

Morris, R. G. M. (1981). Spatial localization does not require the presence of local cues. Learning and Motivation, 12, 239-260.

Nilsson, M., Perfilieva, E., Johansson, U., Orwar, O., \& Eriksson, P. S. (1999). Enriched environment increases neurogenesis in the adult rat dentate gyrus and improves spatial memory. Journal of Neurobiology, 39, 569-578.

Nithianantharajah, J., \& Hannan, A. J. (2006). Enriched environments, experience-dependent plasticity and disorders of the nervous system. Nature Reviews, 7, 697-709.

Parker, S. (1990). Grzimek's encyclopedia of mammals (Vol. 3). New York: McGraw-Hill.

Pearce, J. M. (2009). The 36th Sir Frederick Bartlett lecture: An associative analysis of spatial learning. Quarterly Journal of Experimental Psychology, 62, 1665-1684.

Pham, T. M., Söderström, S., Winbald, B., \& Mohammed, A. (1999). Effects of environmental enrichment on cognitive function and hippocampal NGF in the non-handled rats. Behavioural Brain Research, 103, 63-70.

Rodríguez, C. A., Chamizo, V. D., \& Mackintosh, N. J. (2011). Overshadowing and blocking between landmark learning and shape learning: The importance of sex differences. Learning and Behavior, 39, 324-335.

Rodríguez, C. A., Chamizo, V. D., \& Mackintosh, N. J. (2013). Do hormonal changes that appear at the onset of puberty determine the strategies used by female rats when solving a navigation task? Hormones and Behavior, 64, 122-135.

Rodríguez, C. A., Torres, A., Mackintosh, N. J., \& Chamizo, V. D. (2010). Sex differences in the strategies used by rats to solve a navigation task. Journal of Experimental Psychology: Animal Behavior Processes, 36, 395-401.

Roof, R. L., \& Stein, D. G. (1999). Gender differences in Morris water maze performance depend on task parameters. Physiology and Behavior, 68, 81-86.

Sandstrom, N. J., Kaufman, J., \& Huettel, S. A. (1998). Males and females use different distal cues in a virtual environment navigation task. Cognitive Brain Research, 6, 351-360.

Saucier, D. M., Green, S. M., Leason, J., MacFadden, A., Bell, S., \& Elias, L. J. (2002). Are sex differences in navigation caused by sexually dimorphic strategies or by differences in the ability to use the strategies? Behavioral Neuroscience, $116,403-410$.

Sherwin, C. M. (1998). Voluntary wheel running: A review and novel interpretation. Animal Behavior, 56, 11-27. 
Silverman, I., Choi, J., \& Peters, M. (2007). The hunter-gatherer theory of sex differences in spatial abilities: Data from 40 countries. Archives of Sexual Behavior, 36, 261-268.

Simpson, J., \& Kelly, J. P. (2011). The impact of environmental enrichment in laboratory rats-Behavioral and neurochemical aspects. Behavioural Brain Research, 222, 246-264.

Sutherland, J., \& Hamilton, D. A. (2004). Rodent spatial navigation: at crossroads of cognition and movement. Neuroscience and Biobehavioral Reviews, 28, 687-697.

Treit, D., \& Fundytus, M. (1988). Thigmotaxis as a test for anxiolytic activity in rats. Pharmacology Biochemistry and Behavior, 31, 959962.

Uda, M., Ishido, M., Kami, K., \& Masuhara, M. (2006). Effects of chronic treadmill running on neurogenesis in the dentate gyrus of the hippocampus of adult rat. Brain Research, 1104, 64-72.

van Praag, H., Christie, B. R., Sejnowski, T. J., \& Gage, F. H. (1999a). Running enhances neurogenesis, learning, and long- term potentiation in mice. Proceedings of the National Academy of Sciences of the United States of America, 96(23), 13427-13431.

van Praag, H., Kempermann, G., \& Gage, F. H. (1999b). Running increases cell proliferation and neurogenesis in the adult mouse dentate gyrus. Nature Neuroscience, 2, 266-270.

Vashro, L., \& Cashdan, E. (2015). Spatial cognition, mobility, and reproductive success in northwestern Namibia. Evolution and Human Behavior, 36, 123-129.

Voyer, D., Voyer, S., \& Bryden, M. P. (1995). Magnitude of sex differences in spatial abilities: A meta-analysis and consideration of critical variables. Psychological Bulletin, 117, 250-270.

Williams, C. L., Barnett, A. M., \& Meck, W. H. (1990). Organizational effects of early gonadal secretions on sexual differentiation in spatial memory. Behavioral Neuroscience, 104, 84-97.

Williams, C. L., \& Meck, W. H. (1991). The organizational effects of gonadal steroids on sexually dimorphic spatial ability. Psychoneuroendocrinology, 16, 155-176. 